\title{
Desarrollo territorial rural: ¿una oportunidad para incluir a los pobres en el desarrollo?
}

\section{Cristobal Maldidier"}

\section{Recibido: junio de 2012/ Aceptado: julio de 2012}

El funcionamiento de los proyectos de desarrollo de la cooperación internacional no ha sido consistente con la búsqueda de rutas de desarrollo para los sectores pobres y vulnerables de las áreas rurales. Hoy en día, un nuevo enfoque llamado de desarrollo territorial rural pretende ofrecer posibilidades nuevas para agilizar un giro en las prácticas de los proyectos. ¿Será solamente una nueva etiqueta para designar viejas recetas o constituye realmente un nuevo concepto? ¿En qué medida y bajo qué condiciones este enfoque ofrece nuevas oportunidades para fomentar un desarrollo que incluya a los grupos sociales más desfavorecidos de las áreas rurales? En la primera parte del artículo se revisitan las razones por las cuales los proyectos de desarrollo, aunque sean "participativos", no logran beneficiar a los grupos vulnerables, recurriendo al análisis de los juegos de actores en las intervenciones de desarrollo, tal como lo ha abordado la antropología del desarrollo. En una segunda parte se analiza cómo el enfoque de "desarrollo territorial", al reconocer el protagonismo de los actores locales en manejar su propio futuro y la necesidad de negociar con ellos los proyectos, representa un avance que permite mejorar las prácticas de los proyectos de la cooperación internacional. Sin embargo, sin abrir específicamente los espacios para que los grupos vulnerables puedan negociar sus propias agendas de desarrollo, y sin ayudar a que estos grupos aumenten sus capacidades de iniciativa local, de organización y de representación, el desarrollo territorial seguirá siendo tan excluyente como siempre. Se concluye cómo le toca a las ONG y aquellos organismos de desarrollo comprometidos - por sus valores y su historia - con los sectores excluidos del campo, consolidar sus alianzas y compromisos con este sector, y re-politizar su agenda, alejándose del carácter a menudo técnico y "despolitizado" de los proyectos de desarrollo.

Palabras clave: proyectos de desarrollo / desarrollo territorial / grupos vulnerables / concertación 


\section{Introducción}

En Nicaragua, el desarrollo rural de las últimas dos décadas está basado en gran medida en la gran producción agropecuaria, principal fuente de crecimiento de la economía del país. Este modelo apunta en la dirección de una concentración cada vez mayor de los recursos en pocas manos y ha contribuido a un aumento de las desigualdades sociales en el país. A pesar del crecimiento agrícola y del fuerte dinamismo de algunas cadenas (carne y lácteos, por ejemplo), muchos pequeños productores quedan excluidos del desarrollo. Se estima que cerca del $70 \%$ de la población rural sigue viviendo en condiciones de pobreza (Grisgby, 2010).

Los cuantiosos recursos de la cooperación internacional, la implementación de programas específicos de lucha contra la pobreza, así como los numerosos proyectos de desarrollo en áreas rurales que pretendían reducir la pobreza rural, no han tenido mayor éxito. Las contribuciones posiblemente más eficaces para reducir la pobreza han venido, paradójicamente, de los mismos pobres, al menos de aquellos más jóvenes que han optado - frente a la falta de acceso a los recursos productivos y a la escasez de oportunidades de empleo - por la migración fuera del país, enviando remesas a sus familias (Perez, 2010).

Entre las explicaciones comunes de este fracaso se menciona el hecho de que estos proyectos y políticas no han logrado atacar las causas estructurales de la pobreza rural, en particular las desigualdades en el acceso a la tierra y a los recursos productivos. Asimismo, en el marco del modelo neoliberal, el Estado, debilitado, ha perdido su capacidad de orientar la economía e implementar políticas públicas eficaces. Otra explicación, partiendo de la idea de que este sector más vulnerable del área rural merece ser subsidiado (en productos alimenticios, educación, salud, etc.), apunta a la falta de recursos financieros. En este artículo, inspirado en la sociología y la antropología del desarrollo, nos interesamos más bien, en la manera en que operan o funcionan los proyectos de desarrollo, vistos como un juego de actores en una arena específica.

Un nuevo enfoque, llamado de desarrollo rural territorial, pretende superar las tradicionales y fracasada políticas de desarrollo rural, buscando cómo crear nuevas sinergias entre los actores locales y las redes institucionales. En Nicaragua también está en marcha un cambio de la institucionalidad pública para el desarrollo rural desde una visión sectorial hacia este enfoque territorial. El desarrollo territorial, además de articular la economía del territorio a los mercados dinámicos externos, se plantea mejorar la institucionalidad local, o sea las reglas de interacciones entre los actores locales entre sí, y entre ellos y los agentes externos. Éste ha sido definido como un "proceso de transformación productiva e institucional en un espacio rural determinado cuyo fin es reducir la pobreza rural" (Shejtman \& Berdegué, 2004, p. 11).

El desarrollo territorial, ¿será una nueva palabra para designar viejas recetas o constituye realmente un nuevo concepto? ¿En qué medida y bajo qué condiciones este enfoque ofrece nuevas oportunidades de fomentar un desarrollo que incluya a los grupos sociales más desfavorecidos de las áreas rurales?

Designaremos aquí a estos sectores sociales desfavorecidos con las palabras 
"pobres" o bien "grupos vulnerables", o sea, grupos socialmente dominados, excluidos del acceso a los recursos porque "terminan en el lado perdedor en las múltiples negociaciones sobre el acceso a recursos y oportunidades" (Bastiaensen, De Herdt \& D'Exelle, 2005, p. 981).

En la primera parte revisitaremos las razones por las cuales los proyectos de desarrollo no logran beneficiar a los grupos vulnerables, recurriendo al análisis de los juegos de actores en las intervenciones de desarrollo. En una segunda parte veremos cómo este enfoque es útil para entender los cambios que posibilita el desarrollo territorial y discutiremos en qué medida el enfoque de desarrollo territorial puede ser exitoso en desatar el desarrollo para los más pobres. Nos apoyaremos en algunos ejemplos tomados de Nicaragua, y más específicamente, basados en la experiencia de Nitlapán, el Instituto de Investigación y Desarrollo de la UCA, en una de sus regiones de intervención, el centro norte del país.

\section{Algunos límites de los proyectos "tradicionales" para fomentar un desarrollo incluyente}

Revisaremos primero algunos elementos críticos del funcionamiento "normal" de los proyectos de la cooperación internacional para tratar de explicar cómo fallan en su intento de cambiar la situación de los pobres.

\subsection{La rigidez de los proyectos en el marco de la cooperación internacional}

Una primera crítica comúnmente mencionada se relaciona con el formato "proyecto". Clásicamente, se define un proyecto como la movilización programada de determinadas actividades y medios para lograr, en algún plazo determinado, objetivos específicos. Este modelo linear y racional está probablemente más adaptado para prever la ejecución de obras físicas que la realización de procesos complejos de cambio social (el desarrollo rural).

En el caso de la cooperación internacional, el proyecto se enmarca en un contexto institucional específico que son las "cadenas de la ayuda externa". Estas cadenas son a menudo largas, de tres a cinco eslabones: los financiadores externos (a veces con delegación en el país); el organismo de desarrollo (por ejemplo una ONG capitalina), a veces con su sucursal local; los actores del territorio, a quienes va dirigido el proyecto, más algún intermediario local. Entre estos eslabones existe una distancia, no solamente física o geográfica sino también social y cultural, que dificulta el diálogo y la comprensión mutua a pesar de que varios eslabones intermedios tengan una función implícita de mediadores. Debido, además, a un funcionamiento a menudo unilateral y poco recíproco de estas cadenas, el proyecto resulta a menudo ser insuficientemente flexible para adaptarse a los contextos locales en los cuales se implementa. Los beneficiarios, los últimos eslabones, pueden difícilmente reorientar su contenido, sus procedimientos.

La lógica de este sistema, que parece ofrecer soluciones ya pre-establecidas en cualquier parte sin preocuparse por si calzan o no, ha sido resumida irónicamente como la de "buscar problemas para las soluciones" (Naudet, 1998). El enfoque 
llamado de la "mirada invertida" (Zellweger, citado por Soriano \& Tapia, 2005), que analiza los procesos de decisión, informes y flujo de dinero de la cooperación a la luz de los procesos normales de compraventa en el mercado, destaca cómo los eslabones intermedios responden sobre todo a los modelos de los financiadores externos.

Una de la consecuencia es que no queda más remedio para los eslabones intermedios y finales de estas cadenas de desarrollo que buscar cómo manipular las reglas del proyecto para adaptarlas a su contexto y sus estrategias propias. $\mathrm{O}$ sea, "entrar con lo de ellos y salir con lo nuestro". Estas estrategias permiten la realización de algunos ajustes que aumentan la pertinencia o la eficiencia de los proyectos, pero son también estrategias oportunistas donde individuos o grupos logran desviar los objetivos sociales de los proyectos para agendas particulares.

El hecho de que, en las últimas décadas, los proyectos hayan reivindicado ser "participativos" no ha cambiado tanto la situación. Esta "participación" busca a menudo cómo hacer más eficaz al proyecto pero sin dejar un verdadero espacio para que sean modificados sus contenidos y sus modos de operar. Ha predominado una lógica instrumental de la participación (Chambers, 2005). Los "diagnósticos" participativos, que tenían como propósito hacer emerger las verdaderas "necesidades" de la gente, son en realidad orientados por alguna oferta implícita, asemejándose a fin de cuentas a cierta modalidad de manipulación de las poblaciones locales (Mosse, 2000).

Esta rigidez de los proyectos de cooperación internacional se ha agudizado fuertemente con la agudización del manejo "gerencial" de estos proyectos, que se ha traducido en una tendencia de racionalización y estandarización de los formatos, y en la hegemonía creciente de los procedimientos administrativos y financieros en todo el "ciclo del proyecto". Ha fortalecido aún más el fenómeno de la "mirada invertida" mencionado más arriba. Herramientas como el marco lógico, que se han vuelto casi universales, son un reflejo de la manera en que se concibe y se regula este mundo específico del desarrollo.

Cuando los proyectos se multiplican en un mismo espacio, aumentan los efectos perversos. Es común encontrar, para los miembros de una misma comunidad o cooperativa, que las reglas de acceso a los recursos varíen de un proyecto a otro en cuanto a su carácter devolutivo o no (donación o reembolsable), o en cuanto a los beneficiarios meta (reglas de selección). Esta competencia entre proyectos propicia al final de cuentas una segmentación social (véase, por ejemplo, Mendoza, 2010).

\subsection{La exclusión de los grupos vulnerables de los proyectos}

La mayoría de los recursos que acompañan los proyectos se quedan en las manos de los grupos dominantes y más acomodados de las comunidades o de los territorios, y los pobres no logran más que "migajas". Son numerosos los ejemplos. A nivel de comunidades, se observa con frecuencia cómo algunos individuos, bautizados "líderes de la comunidad" por los proyectos, logran consolidar su posición social y económica en dicha comunidad gracias a los proyectos, apropiándose eventualmente de una parte de estos recursos (Mosse, 2001). Lejos de la imagen de comunidad unida y del consenso comunitario que aquel líder trata de ofrecer al visitante de paso y a los actores externos, las comunidades están a menudo divididas entre varias 
redes sociales - cada cual con su(s) líder(es) - que compiten entre ellas (Molenaers, 2006). Además, en el seno de cada una de ellas dominan relaciones de dependencia, explotación y exclusión

A nivel de un territorio entero es igual. Por ejemplo, en los municipios de Matiguás y Río Blanco, se puede constatar cómo los ganaderos medianos y grandes, quienes controlan las estructuras locales de poder, lograron captar los abundantes recursos de la cooperación internacional (proyecto "Proderbo" de la Unión Europea en la década de los 90, proyecto "Fondeagro" en la década de los 2000), para fortalecer un modelo de desarrollo basado en grandes fincas lecheras vinculadas a la industria nacional. Los más pequeños y los pobres tuvieron que irse montaña adentro (Mendoza, Fernández \& Hernández, 2011).

Estos ejemplos levantan una interrogación importante: ¿no serian las modalidades mismas de las intervenciones de los proyectos las que crearían una exclusión de los más pobres? Mencionemos dos rasgos característicos de las modalidades de operar de los proyectos.

El primero es que los requisitos que imponen los proyectos se basan en un "mundo" jurídico e institucional que está ligado al Estado moderno pero que es, muchas veces, ajeno a las prácticas y las representaciones de los más vulnerables de la comarca. Sólo algunos, en general acomodados, son los que pueden maniobrar con facilidad entre los dos marcos: las reglas formales estatales y las reglas informales que gobiernan la convivencia local (Bastiaensen, D’Exelle \& Famerée, 2006). Por estas razones, los respaldos "oficiales", como los títulos de propiedad exigidos por las microfinancieras, alejan a los más vulnerables de la misma manera que imponer a las organizaciones formales (cooperativas, comités, etc.) como canal obligatorio entre las poblaciones locales y los financiadores (Gómez \& Ravnborg, 2006).

En segundo lugar, la "oferta" de los proyectos no se adecua generalmente a las estrategias de vida de los grupos más vulnerables (Bastiaensen et al., 2011). Puede ser una oferta diseñada por hombres y promovida en las comunidades por técnicos hombres, que no responde a los intereses de las beneficiarias potenciales mujeres, o simplemente no las considera como beneficiarias posibles. Es común observar cómo los técnicos de proyectos, al ofertar sus llamados "paquetes técnicos", orientan inconscientemente sus acciones hacia ciertos estratos de productores (a menudo acomodados) que son los únicos capaces y deseosos de adoptar las tecnologías que ellos promueven. En cambio, estas tecnologías resultan ser demasiado arriesgadas para los pobres por sus estrategias anti-riesgos; las puedan adoptar, salvo en caso de que estén subsidiadas.

Los proyectos argumentan que responden a una "demanda" de los beneficiarios, sin darse cuenta de que en realidad las poblaciones demandan comúnmente lo que los proyectos ofertan porque saben que muy raras veces estos proyectos son capaces realmente de hacer caso a sus demandas reales. Al aceptar el paquete ofertado, aunque no sea muy adaptado ni muy útil para su proceso de acumulación, no se arriesgan tanto porque saben que hay posibilidad de que sea subsidiado, y además estrechan lazos de confianza con una institución externa que potencialmente puede traer nuevos proyectos y nuevos beneficios. 


\subsection{El proyecto, una intervención externa en medio de competencia y negociaciones entre actores}

Las ciencias sociales han propuesto marcos conceptuales que ayudan a entender las realidades descritas más arriba. Las intervenciones de desarrollo se conciben como "un proceso continuo, construido socialmente y de manera negociada (entre actores involucrados de manera directa e indirecta) en lugar de la simple ejecución de un plan de acción previamente especificado con resultados esperados" (Long, 1999 citado en Bastianensen et al, 2010). Más precisamente, es una intervención externa en sistemas sociales dinámicos que consiste en transferir recursos a distintos grupos locales, quienes van a intentar captarlos para favorecer sus propias estrategias o, al contrario, para neutralizar efectos negativos (Olivier de Sardan, 2005). Estos recursos son de tipo material y económico (crédito, herramientas o animales, etc.), pero suelen ser también de carácter inmaterial, como son los saberes (técnicos, organizacionales), o de tipo simbólico, como es el reforzamiento del prestigio o del poder de un individuo, de un grupo.

Esta "exterioridad" de los proyectos en relación a los sistemas sociales en los cuales intervienen es siempre problemática. ¿Cómo, por ejemplo, seleccionar a los más pobres y evitar que algunos menos pobres aprovechen la falta de conocimientos del proyecto sobre su acceso a los recursos para hacerse pasar por pobres? ¿Cómo mejorar los conocimientos parciales y fragmentados sobre las realidades locales (más allá de los siempre parciales "diagnósticos" iniciales) para evitar errores en la ejecución del proyecto? Por esto las instituciones que promueven estos proyectos, y las ONG en particular, buscan cómo reducir esta brecha a través de su arraigo local. Sus promotores o técnicos locales, que son los principales enlaces entre el proyecto y las comunidades locales, por su origen social y geográfico tienen un rol clave en este intento de arraigar al proyecto, como ha sido ilustrado para el caso de micro financieras (d'Exelle, Bastiaensen \& Alemán, 2003). Mosse (2000) habla de colusión entre promotores de desarrollo y algunos grupos dominantes, en donde los primeros garantizan la ejecución de los proyectos y el cumplimiento de las metas a cambio de sesgos y ventajas para ellos. Esta "remuneración" (material pero también política o simbólica) que compensa la inversión individual de muchos dirigentes locales en el quehacer de los proyectos ha de ser suficientemente explícita y transparente para ser legítima en la comunidad.

Todo proyecto interviene en un juego social local complejo, una arena sociopolítica donde se confrontan actores con lógicas heterogéneas y poderes distintos. En torno a los recursos del proyecto, se da una situación de competencia y de negociaciones entre distintos grupos e individuos. Esta negociación constituye una "participación escondida a los proyectos" (Chauveau, 1995), o sea, una participación discreta de los diferentes grupos de actores, de la cual el personal del proyecto no se da necesariamente cuenta y que tiene lugar paralelamente a las modalidades de participación "oficial" promovidas por el proyecto. Precisemos que estos grupos no corresponden a categorías fijas sencillas, como son las tipologías de productores o categorías generales como "las mujeres", "los jóvenes", sino que son redes sociales o "grupos estratégicos" (reuniendo individuos que se unen en torno a algún interés específico común ligado a alguna esfera específica). 
Los pobres o los grupos vulnerables no son grupos que se pueden caracterizar por indicadores cuantitativos precisos y claramente identificables. La pobreza y la vulnerabilidad se inscriben en un proceso de exclusión del acceso a los recursos, y se trata de un fenómeno dinámico y político. Los proyectos que buscan cómo beneficiar a los más pobres alteran el sistema de regulación de las desigualdades y enfrentan la resistencia de la estructura local del poder. Cuando un proyecto otorga una vaca al crédito o en leasing ${ }^{1}$ a una familia pobre, o cuando busca cómo facilitar el acceso a la tierra, interviene de manera más o menos indirecta en una relación de tipo cliente-patrón: el patrón pierde cierto control sobre una fuente de mano de obra. Beneficiar específicamente a los más vulnerables significa, entonces, imponer una regla adicional en un sistema de reglas existente sobre el cual el proyecto tiene poco control.

No cabe, sin embargo, ningún fatalismo que consista en creer que siempre los más pudientes serán los que saldrán ganando de cualquier proyecto. Los grupos meta, aun los más pobres, no son "víctimas" sino actores con cierto margen de maniobra, que saben usar estrategias "solapadas" para lograr sacar alguna ventaja de los proyectos. Lograr ampliar los márgenes de maniobra de los más vulnerables es parte de los retos de los proyectos, pero no es una tarea imposible.

\section{3. ¿Contribuyendo a un proyecto de territorio que incluya a los más pobres?}

\subsection{El enfoque del desarrollo territorial y las implicaciones para los proyectos}

En los últimos años, una perspectiva nueva en los proyectos de desarrollo rural ha surgido en torno a la dimensión territorial. A diferencia de lo que vimos más arriba, el territorio no es aquí un simple espacio físico "objetivamente existente y delimitable, por un puro ejercicio técnico ex ante en virtud de una u otra variable o conjunto de variables, físicas o económicas” (Schejtman \& Berdegué, 2004, p. 33) sino que se concibe como "un conjunto de relaciones sociales que dan origen y a la vez expresan una identidad y un sentido de propósito compartidos por múltiples agentes públicos y privados" (Schejtman \& Berdegué, 2004, p. 30).

En él los actores establecen relaciones de coordinación para responder a determinado problema económico o productivo, o incluso para hacer emerger un proyecto para su territorio, o sea, un proyecto que armoniza a los diferentes proyectos individuales y colectivos de los habitantes en torno a algunas actividades económicas predominantes que estén basadas en las riquezas y en los recursos específicos del territorio.

El territorio es entonces una construcción social, y se construye en estos procesos de coordinación de los actores, movilizados en torno a objetivos comunes.

1 El leasing se refiere a todo contrato de arrendamiento de bienes donde una institución cede su usufructo, durante un plazo de tiempo determinado contractualmente, a un tercero quien, a cambio, está obligado a pagar un monto periódico. Al concluirse el plazo, el usuario tiene dos opciones: la de comprar el bien por su valor residual o la de devolverlo. 
Es una "realidad emergente", producto de procesos de auto-organización del sistema social. Sin embargo, no se trata de un avatar del enfoque de "desarrollo local", que emergió en la década de los 80 en oposición al verticalismo del Estado desarrollista y basado en una movilización social de la comunidad como fuente de cambio exclusiva en materia de desarrollo.

El desarrollo territorial reconoce, en efecto, que el territorio es un lugar privilegiado de interacciones entre actores, proyectos y políticas sectoriales, y de ahí la importancia de abordar las relaciones entre instituciones estatales, gobierno local, comunidades, empresas privadas, etc. Asimismo, el territorio y sus habitantes no es un espacio "aislado": existen redes de relaciones que unen a individuos y grupos situados a diferentes niveles territoriales (micro, local, municipal, departamental, nacional e internacional). Por lo tanto, la clave del desarrollo territorial es la articulación entre las dinámicas a estos distintos niveles, lo cual permite superar la distinción habitual entre proyectos top-down y proyectos bottom-up.

¿Que consecuencias esto tiene para los proyectos? A diferencia del enfoque participativo, que tiende a limitar la intervención a algunas categorías de actores locales en función de sus objetivos, este enfoque busca cómo incluir un abanico más amplio de actores locales. Entre ellos está el gobierno local, que aspira a fortalecer sus espacios para definir e implementar su estrategia de desarrollo municipal. Están también los representantes locales del Estado, que tienen que buscar innovadoras maneras de adaptar localmente las políticas nacionales. Están finalmente los actores organizados de la sociedad civil (cooperativas, gremios, asociaciones locales), los privados etc. $\mathrm{Al}$ asumir esta diversidad de actores, los proyectos han de abandonar toda idea de consenso ficticio en torno a los caminos de desarrollo, y aceptar la existencia de diferencias de intereses y de contradicciones más o menos conflictivas entre los distintos actores. Le toca a la intervención externa incrementar, en una perspectiva de largo plazo, las capacidades de los actores de concertarse, de coordinarse, de participar en dinámicas colectivas, en un ámbito de acción colectiva.

Asimismo, el territorio deja de ser un "territorio de proyectos", donde el Estado y cada ONG ejecutan sus proyectos, llevan a cabo sus programas, y donde los habitantes son considerados simples beneficiarios de ayudas (buscan cómo "agarrarse a un proyecto") según las oportunidades que aparezcan, sin responder a ningún plan o lógica explícita. El enfoque del desarrollo territorial, al reconocer el protagonismo de los actores locales en manejar su propio desarrollo, legitima el hecho de que los proyectos han de ser negociados con estos mismos actores locales. Esta negociación no apunta a realizar algunos ajustes para optimizar la ejecución del proyecto tal como ha sido predefinido, sino que abre a negociación, en la arena local, los campos mismos de intervención, los contenidos concretos, los grupos meta, las reglas y procedimientos de implementación. Los actores locales (incluyendo a los vulnerables, como lo veremos más abajo) han de tener este poder de modificar el quehacer de los proyectos. No significa que van a desaparecer por completo aquellos juegos discretos de negociación, sino que los proyectos van a intentar sacarle provecho a las capacidades locales de confrontarse, de llegar a convergencias, aunque sean parciales y puntuales. Una nueva era de los proyectos ha entonces de abrirse: después de los proyectos participativos, con las ambigüedades que vimos más arriba, el desarrollo territorial abre quizás el tiempo de la negociación (Leeuwis, 2000). 


\section{2. ¿Qué lugar ocupan los grupos vulnerables en los proyectos con enfoque de desarrollo territorial?}

¿De qué manera y bajo qué condiciones este enfoque de desarrollo territorial facilita o no un desarrollo más incluyente? ¿Acaso las redes de notables locales no van, a nivel del territorio, a seguir monopolizando las vías y recursos de desarrollo, en detrimento de los intereses de los grupos vulnerables? El territorio, ¿no es acaso "un paisaje con vías de amplios carriles para algunos sectores sociales, con estrechas veredas para otros y con profundas quebradas y malos caminos (en un sentido real y simbólico) para los más vulnerables"? (Bastiaensen et al., 2011, p. 22).

Varios autores ubican a la concertación como eje central de la nueva institucionalidad que requiere el desarrollo territorial. Por "concertación" se entiende un proceso de diálogo y de búsqueda de acuerdos con el objetivo de desembocar en propuestas, proyectos u orientaciones aceptadas por todas las partes ${ }^{2}$. Una hipótesis es que a pesar de que las estructuras sociales locales están marcadas por relaciones de dominación de tipo patrón-cliente y por una fuerte segmentación social, existe también en los territorios un tejido más o menos denso de relaciones horizontales de cooperación vecinal o familiar, y capacidad para que grupos heterogéneos o coaliciones de diferentes actores logren coordinarse y empujar iniciativas comunes (Gómez, 2001).

Shejtman y Berdegué (2004, p. 11) plantean combinar exitosamente dos elementos distintos: "estimular y facilitar la interacción y la concertación de los actores locales entre sí y entre ellos y los agentes externos relevantes, $\underline{\mathrm{e}}^{3}$ incrementar las oportunidades de que la población pobre participe del proceso y de sus beneficios". Sin embargo, ¿qué logros pueden alcanzar realmente los grupos vulnerables en estos procesos de concertación? ¿los grupos dominantes estarán realmente en disposición de soltar una parte de su poder para que los más vulnerables puedan orientar el proyecto y fortalecer así sus propias rutas de desarrollo?

Es de temer que sin la apertura de espacios para que esta negociación se pueda preparar y realizar de manera favorable para los más vulnerables y sin apoyo especifico a estos sectores, la concertación termine siendo en definitiva una manera para los grupos dominantes de reducir el riesgo de una posible oposición de los grupos vulnerables.

La responsabilidad de las intervenciones de desarrollo es que las demandas de los grupos vulnerables se formulen y se oigan en las arenas locales, que las modalidades concretas bajo las cuales se realizan los procesos de concertación incluyan a estos grupos y que al menos una parte de los recursos se puedan orientar de manera transparente, sin paternalismo ni clientelismo hacia estas rutas de desarrollo "alternativas". Paralelamente y progresivamente, se trata de mejorar la capacidad de estos sectores de asociarse, organizarse y mejorar su representación y su poder político en las estructuras de poder territoriales (municipio, comité local, etc.), cooperativas

\footnotetext{
2 Este proceso de concertación suele tener una faceta informal y, por naturaleza, poco visible para un ojo externo, así como una faceta "institucionalizada" a través de instancias formales, puntuales (en el marco de un proyecto) o permanentes (consejo de desarrollo municipal, por ejemplo).

3 El subrayado es nuestro.
} 
o gremiales ${ }^{4}$. Es en el marco de dicho proceso de construcción de largo plazo que son aceptables convergencias parciales de intereses con los actores pudientes y consensos puntuales a nivel local, con la condición de que sean favorables a las estrategias de los grupos vulnerables. Recurriendo a la imagen conocida del boxeo, corresponde a la técnica del "golpe y amarre" (P. Marchetti, comunicación personal, 1992)5.

Para ello, las ONG y especialmente las comprometidas con los pobres han de fortalecer sus alianzas estratégicas con estos sectores y sus organizaciones, aunque esta posición no sea compatible con la posición en principio más "neutral" del animador/mediador de procesos de concertación. Este posicionamiento constituye un retorno a los enfoques originales desarrollados por Paulo Freire (1972), enfoque de naturaleza política, donde los interventores exteriores se consideraban como catalizadores de dinámicas de grupo para que los actores locales "marginalizados" reforzaran su autoestima y su poder político, y progresivamente esto desembocara en una planificación autónoma de la población.

\subsection{Una ilustración: el caso del desarrollo excluyente de la vía láctea y los caminos alternativos}

Los cambios experimentados por las viejas fronteras agrícolas ganaderas de Matiguás y Río Blanco nos servirán de ilustración. Flores et al. (2011) han puesto de relieve, en una detallada investigación, cómo en los últimos 15 años la leche fresca se ha vuelto el eje aglutinador del desarrollo territorial. Esto se ha logrado gracias a una coordinación bastante eficaz de diversos actores locales entre sí (productores, gremio ganadero, centros privados o cooperativas de acopio, transportistas, gobierno local, etc.) y con actores externos (agroindustria en Managua, instituciones públicas, empresas de servicios, cooperación internacional, etc.). Para ello, fondos públicos del Estado central (para el mejoramiento de la infraestructura vial y de energía) se han combinado con inversión privada, recursos de la cooperación internacional y fondos del gobierno local.

Ha emergido entonces un territorio lechero que ha logrado imponer progresivamente su hegemonía sobre el espacio regional, cruzando a tres municipios (Muy-Muy, Matiguás y Rió Blanco). Esta "vía láctea" tiene la forma de un pulpo, cuyos brazos son la red de caminos de todo tiempo, los cuales se van multiplicando y extendiendo. El proceso de extensión es sencillo: la clave es el camino de todo tiempo que permite conectarse con un camino existente y que rentabiliza las inversiones de un centro de acopio de leche, centro neurálgico local de la cadena. El trazado del camino y su hechura supone una movilización colectiva de los productores del micro-territorio ${ }^{6}$ y negociaciones con alcaldías y financiadores externos. Una vez que el camino está hecho, un nuevo grupo de productores puede insertarse en la cadena lechera existente, partiendo de las coordinaciones existentes. Poco a poco la cadena

\footnotetext{
4 A menudo los grupos vulnerables son marginalizados en estas estructuras, y su "invisibilización" tiende a crecer a medida que se elevan en las estructuras, como lo demuestran, por ejemplo, el caso de las cooperativas de segundo o tercer grado (Barrios, 2008).

5 Universidad Campesina, Managua.

6 Un espacio que tiene aproximadamente una anchura que equivale a una hora en mula desde ambos lados del futuro camino.
} 
de leche fresca logra imponerse sobre las cadenas de queso y cuajada.

Sin embargo, este modelo de desarrollo del territorio ha sido muy excluyente desde el punto de vista social. Las exitosas dinámicas colectivas no han logrado incluir a los grupos vulnerables: segmentos de campesinos pobres con tierra (pero poca) y sin capital, mujeres y, particularmente, transformadoras de cuajada (Flores et al., 2011). Su situación se ha deteriorado, provocando un empobrecimiento local, el repliegue en las partes menos accesibles del territorio o la migración hacia la profundidad de la montaña. El proceso social y espacial histórico del avance de la frontera agrícola (Maldidier, 2004) se ha reactivado, entonces, bajo modalidades nuevas debido a la especificidad de la leche, aunque con los mismos resultados de "avance en cascada" amarrados a la polarización social. En definitiva, los grupos vulnerables, poco organizados, poco presentes en las estructuras de poder local y sub representados en las instancias formales de concertación, han sido invisibilizados en los procesos de transformación rural.

Otras vías de desarrollo hubiesen sido quizá posibles (y siguen siéndolo, aunque hoy en día probablemente sea en otras partes del territorio) si se habían apoyado las pocas iniciativas más o menos colectivas de los grupos vulnerables. En efecto la conflictividad social que se ha incrementado fuertemente en la región, traduce también la existencia de varios focos de resistencia a este modelo de desarrollo lechero (Mendoza et al., 2011). Las intervenciones externas hubieran podido (pueden) darse como meta de apoyar sistemáticamente a los grupos vulnerables en sus intentos más o menos colectivos, estructurados y exitosos de defender las rutas de desarrollo que más les convienen.

Las cadenas de queso y de cuajadas particularmente, en mayor medida ligadas a estos grupos vulnerables, se pueden potenciar, con un enfoque de cadena de valor. Esto implica fomentar los encadenamientos locales promoviendo la cooperación entre un tejido de pequeñas estructuras de producción y MIPYMEs de procesamiento y comercialización en torno a un producto de calidad destinado a algunos segmentos de compradores urbanos. En un segundo momento se podrá explorar si estos productos agroalimentarios pueden ser identificados a través de una especificidad, una fama o una imagen territorial, y entonces apoyar para que las organizaciones de productores se involucren en estrategias territoriales y de construcción de signos de calidad vinculados al origen geográfico ${ }^{7}$. La coexistencia de estas cadenas de queso y cuajada con la cadena de la leche, que implicará la búsqueda de formas de regulación adecuadas, pudiera ser una vía que fortalece posiblemente el proyecto territorial colectivo (al menos en la mayor diversificación de la economía del territorio) y la cohesión social en torno a él.

En esta realidad de frontera agrícola, donde la apertura de caminos se acompaña de cambios sociales veloces, cualquier temática prácticamente puede ser aprovechada para empujar dinámicas alternativas en las cuales los grupos vulnerables

7 Ya sea con una Indicación Geográfica (IO), un signo de calidad que se utiliza en productos que tienen un origen geográfico específico y poseen unas cualidades determinadas o una reputación que es atribuible a dicho lugar, o bien una Denominación de Origen (DO), un tipo especial de indicación geográfica que se aplica a productos que poseen una calidad específica derivada exclusiva o esencialmente del medio geográfico en el que se elaboran. 
consoliden su capacidad de negociar los caminos de desarrollo que les conviene: la apertura y el mantenimiento de un camino de todo tiempo, la capitalización de las fincas, la consolidación de los derechos de acceso y uso de la tierra, la promoción de inversiones colectivas de medios y equipos etc.

Sin embargo, no importa en definitiva tanto el tema sino la finalidad: lograr el arranque de una dinámica en que las poblaciones locales, incluyendo a sus sectores más vulnerables, inicien una movilización, y se consolide un proceso colectivo de interacciones y negociaciones con los otros niveles territoriales y otros actores institucionales. Esto implica largos y múltiples procesos para facilitar reflexión colectiva y movilización en las comunidades, ayudar a que los grupos encuentren sus propias soluciones y aumenten sus capacidades de iniciativa local, de organización y de representación.

\section{Conclusiones}

El funcionamiento de los proyectos de desarrollo de la cooperación internacional no ha sido consistente con la búsqueda de rutas de desarrollo para los sectores pobres y vulnerables de las áreas rurales que siguen siendo considerados implícita o explícitamente más como sujetos de subsidios que como verdaderos actores económicos insertos en un sistema social determinado.

El enfoque de desarrollo territorial ofrece posibilidades nuevas para agilizar un giro en las prácticas de los proyectos. Una intervención de desarrollo que sea negociada y concertada con los actores locales, que fomente y apoye los procesos de concertación, que abra espacios de negociación para favorecer las rutas para los más pobres es, en esencia, una intervención cuyos caminos y resultados son poco previsibles. Le toca a fin de cuentas a todo proyecto, manejar interacciones entre actores, que son relaciones de cooperación más o menos conflictivas y, otras veces, de enfrentamientos más o menos públicos. A medida avanza, se renegocia continuamente el proyecto mismo. Sin duda, el paradigma de "proyecto proceso" convendría más que el del proyecto convencional de corta duración y descontinuados en el tiempo (Mosse, Farrington \& Rew, 1998). Significa considerar que se trata de un aprendizaje colectivo (learning process approach) que asocia a los diferentes actores, y que la planificación ha de ser un sistema flexible en una lógica de proceso de ensayo - error.

Las $\mathrm{ONG}$ y aquellos organismos de desarrollo comprometidos, por sus valores y su historia, con los sectores excluidos del campo, tienen que consolidar sus alianzas y sus compromisos con este sector, y re-politizar su agenda, alejándose del carácter a menudo técnico y "despolitizado" de los proyectos de desarrollo. Si no, es de temer que el desarrollo territorial se vuelva más de lo mismo.

\section{Agradecimientos}

Agradecemos al revisor anónimo de la versión inicial por sus valiosos comentarios. Asimismo, agradecemos al conjunto del personal del Instituto de Investigación Aplicada y Promoción del Desarrollo Local Nitlapán de la UCA por la riqueza de 
los intercambios sobre las prácticas de sus intervenciones que se dieron en el marco de una reflexión sobre el quehacer estratégico de la institución.

\section{Referencias bibliográficas}

Barrios, C. (2008). Marco conceptual sobre desarrollo y promoción de la asociatividad rural con fines económicos. Informe, Apoyo Programático al Sector Agropecuario, Fase 2. APSA II: Managua.

Bastiaensen, J., De Herdt, T. \& D’Exelle, B. (2005). Poverty Reduction as a Local Institutional Process. World Development, 33 (6), 979-993.

Bastiaensen, J., D’Exelle, B. \& Famerée, C. (2006). Arenas políticas alrededor del acceso a la tierra. Un diagnóstico de las prácticas de los derechos de la propiedad en el interior de Nicaragua. Cuadernos de Investigación (26), Nitlapan-UCA: Managua.

Bastiaensen, J., Flores, S., Steel, G., Sosa, C., Martínez, S. \& Alemán, A. (2011). Territorios, actores, y estrategias de desarrollo. Cuaderno de investigación, (40). Nitlapan-UCA: Managua.

D’Exelle, B., Bastiaensen, J. \& Alemán, M. (2003). El microcrédito y el alivio de la pobreza rural en microfinanzas en Nicaragua. En P. Van Dijck, H. Nusselder \& A. Sanders (eds.). Microfinanzas en Nicaragua. Cuadernos del CEDLA, (14), Amsterdam: CEDLA

Chambers, R. (2005). Participation: Review, Reflections, and Future. En R. Chambers (ed.). Ideas for Development. (pp. 97-116). London: Earthscan.

Chauveau, J.P. (1995). Appropriation d'innovations et exclusions de groupes vulnérables dans les projets participatifs de développement rural en Afrique de l'Ouest, Roma: FAO.

Gómez, L. I. (2001). Instituciones y desarrollo rural en Nicaragua. Encuentro, (59), 8-25.

Gómez, L. \& Ravnborg, H. M. (2006). Importancia del capital social para la organización local. Un estudio de exploración sobre capital social en El Castillo, Río San Juan, Nicaragua. Cuaderno de Investigación, (22), NitlapanUCA: Managua.

Grigsby, A. (2010). Qué pasará en las elecciones con una economía tan incierta y tan austera. Envío, (336). Managua.

Flores, S., Barrera, J., Bastiaensen, J., Castro, A., Martínez, S. E. \& Polvorosa, J. C. (2011). Las cadenas de lácteos y su interacción con la dinámica de género: la experiencia en Matiguás y Muy-Muy en Nicaragua. Cuaderno de Investigación, (39), Nitlapan UCA: Managua.

Freire, P. (1972). Pedagogía del oprimido. Buenos Aires: Siglo XXI Argentina Editores.

Leeuwis, C. (2000). Reconceptualizing Participation for Sustainable Rural Development: Towards a Negotiation Approach. Development and Change, (31), 931-959.

Long, N. (1999). The Multiple Optic of Interface -Analysis (Working Tile). Background Paper on Interface Analysis. UNESCO. Recuperado el 30 de junio de 2012, de http://www.utexas.edu/cola/insts/llilas/content/claspo/PDF/ 
workingpapers/multipleoptic.pfd

Maldidier, C. (2004). Agricultural pioneer fronts, the crest of a far-reaching wave. The social and spatial dimension of lowland colonization in Nicaragua. En D. Babin (ed.). Beyond tropical deforestation. From Tropical Deforestation to Forest Cover Dynamics and Forest Development. (pp. 185-192). Montpellier-France: UNESCO CIRAD.

Mendoza, R. (2010). Sistematización de la experiencia de Microleasing en San Juan del Río Coco, Pantasma y Cúa-La Dalia. Informe. Nitlapan-UCA: Managua.

Mendoza, R., Fernández, E. \& Hernández, W. (2011). Detrás de las adversidades están las oportunidades. Diagnóstico Participativo sobre la Conflictividad en el Municipio de Río Blanco. Informe para el programa de Servicios Legales Rurales. NitlapánUCA: Managua.

Molenaers, N. (2006). Vida asociativa y desarrollo local en dos pueblos nicaragüenses. Revista de la CEPAL, (90), 121-133. Recuperado el 1 de junio de 2012, de www. eclac.org/publicaciones /xml/2/27642/LCG2323eMolenaers.pdf

Mosse, D., Farrington, K. \& Rew, A. (eds.). (1998). Development as Process. Concepts and Methods for Working with Complexity. London: Routledge-ODI.

Mosse, D. (2000). Autorité, genre et savoirs: réflexions théoriques sur la pratique du diagnostic participatif en milieu rural. En P. Lavigne Delville, N. E. Sellama \& M. Mathieu (eds.). Les enquêtes participatives en débat. (pp. 359-392). Paris: Karthala.

Mosse, D. (2001). People's knowledge, participation and patronage: operations and representations in rural development. En B. Cooke \& Y. Kothari (eds.). Participation: the new tyranny?. (pp. 16-35). Londres y Nueva York: Zed Books.

Naudet, D. (1998). Finding problems to fit the solutions. Twenty years of Aid to the Sahel. Paris: OECD.

Olivier de Sardan, J-P. (2005). Anthropology and Development: Understanding Contemporary Social Change. Nueva York: Zed Books.

Perez, F. J. (2010). Sin cambios estructurales no habrá una reducción sostenible de la pobreza rural. Envío. (350). Managua.

Schejtman, A. \& Berdegué, J. (2004). Desarrollo Territorial Rural. Serie Debates y Temas Rurales, (1). Santiago, Chile: RIMISP.

Soriano, R. \& Tapia, M. (2005). Pagos en efectivo de la población: un método e instrumento de cualificación de la demanda para mejorar efectos e impactos. La experiencia de pro-rural con recursos Cosude. Sistematización de experiencias Ecuador. Quito: COSUDE. 